\begin{abstract}
We can maximize the impact of scientific conferences by uploading all conference presentations, posters, and abstracts to highly-trafficked public repositories for each content type. Talks can be hosted on sites like YouTube and Youku, posters can be published on Figshare, and papers/abstracts can become Open Access PrePrints.
\end{abstract}

\title{
How to boost the impact of scientific conferences.
}

\author{
Mike Morrison \\ Michigan State University \\ mikeamorrison@gmail.com \\ (corresponding author)
}

\author{
Kelsey Merlo \\ University of South Florida \\ kmerlo@usf.edu
}

\author{
Zach Woessner \\ Michigan State University \\ woessne4@msu.edu
}

Thanks to the heroic efforts of conference administrators across science, most academic conferences scheduled for 2020 have been at least partially translated into a virtual format. Now, it is time to figure out what role online content should have in the future, and how to maximize its engagement and impact.

First, to get in the right mindset, it will help to stop thinking of annual scientific conferences as only updating a subset of attending scientists on what is happening in a field, and start thinking of conferences as being able to update the entire world on what is happening in a field of study - especially all relevant scientists, whether they pay dues for that conference or not.

Figure 1 places the "classic 3" scientific conference content types - Presentations, Posters, and Conference abstracts — on a continuum ranging from 'easy to produce' to 'hard to produce', and from 'small impact' (on just a few people) to 'big impact' (reaching tens or hundreds of thousands of people). 


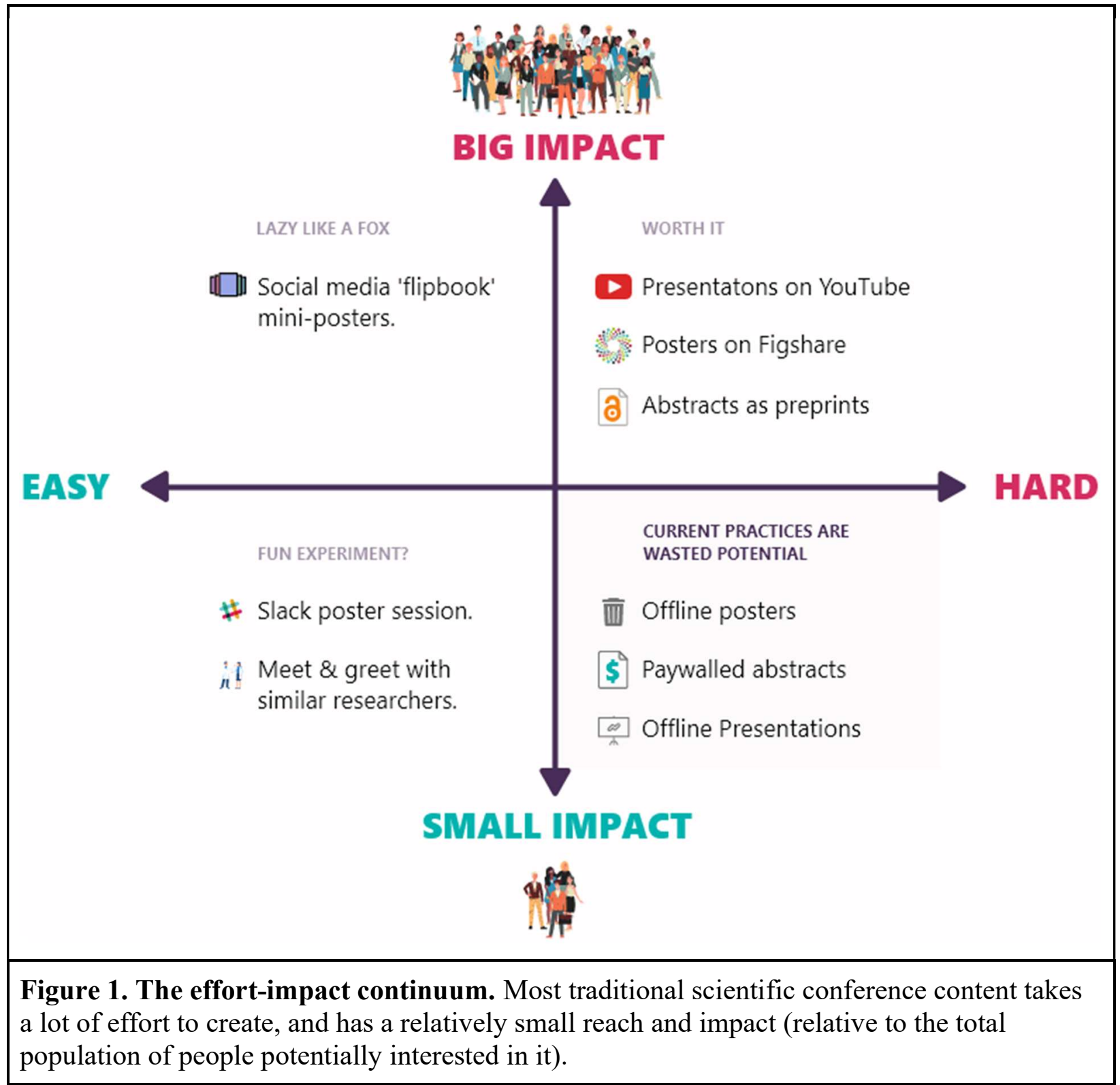

As shown in Figure 1, Presentations and Posters take a great deal of effort to produce, but are only seen by a narrow subset of scientists who pay to attend the conference and also manage to make time for that particular session. As for conference abstracts, it is doubtful whether most are read by anybody besides their peer reviewers and maybe a few persistent researchers who endure the login walls or request copies from the authors. As is typical of the scientific community, we do great work, then put it where nobody can see it.

\section{Record and post presentations on major video sharing sites}

The biggest barrier to increasing the impact of scientific presentations was getting them recorded. The technical barriers to recording scientific talks have been trivial since smartphones 
became ubiquitous (virtual talks are already recorded, and offline talks could be recorded by having a 'designated recorder' in the audience), but until now there has been a big human barrier: scientists have an almost introverted hesitance to record our talks.

Happily, COVID-19 has pushed us all across this threshold, and forced us to get some comfort with the idea of having our talks recorded and posted online. But where is the best place to publish them?

\section{Posting talks behind a paywall is better than nothing}

Having all our talks recorded and posted behind a paywall (or login-wall) on a conference organization's website (as many conferences are doing now during the pandemic) is a huge step towards broader impact, because it helps our talks reach those scientists who attended the conference but missed the talk and those who could not afford the time or money to travel to the conference. But the audience is still limited to members who both pay dues and tolerate the often-clunky conference website.

\section{Sporadic uploading to websites like YouTube and Youku could start a trend towards higher impact}

The impact potential of scientific talks cannot be fully realized until they are posted on major, public video sharing sites like YouTube, because that's where the people are (scientists and otherwise). Sites like YouTube (in the US) and Youku (in China) each receive over one billion views per day (SimilarWeb, 2020). Encouraging scientists to upload their talks to public video sharing sites individually would create sprinklings of high impact, but adoption would likely be patchy as scientists lack experience and comfort with the process, and may be unmotivated to try it because they are inexperienced with how rewarding broad impact can be.

\section{Mass uploading to public video sharing websites would change science and the world}

To ensure that nearly all scientific conference talks are published to popular sites like YouTube and Youku - and give many scientists exposure to the sublime feeling of having a broad impact - we could stop waiting on scientists to do it themselves. Instead, conferences could manage the posting of all recorded talks.

Academic conferences are already abnormal for keeping their conference talks to themselves. In industry, uploading video of all conference events and talks to major video sharing websites is just standard practice. The annual Consumer Electronics Show (CES) in the US generates thousands of YouTube videos every year, covering every talk and event from multiple perspectives, all professionally recorded (CES, 2019).

Science may lack the professional audio-visual staff of a major industry conference, but it makes up for this in sheer industriousness. Many academic conferences since the pandemic have 
demonstrated that they have the human capacity to process and upload hundreds of videos. And where extra manual labor is needed, science's army of undergraduate research assistants (RAs) can be employed to consolidate, edit, and upload the videos. Volunteer RAs who help with this task will be learning about their field's research as they do it.

\section{Posters can have a second life on Figshare}

According to a pilot study we conducted at a major scientific conference, traditional scientific posters received an average of 6.4 visitors, according to presenters' own subjective count (Merlo, Woessner, \& Morrison, 2019). Typically, posters are thrown away after those 6.4 visitors.

Encouraging or requiring poster presenters to upload their final posters to sites like Figshare.com (a website that treats scientific figures as independent, micro-publications) could bring thousands of additional virtual visitors to that poster.

Posters uploaded to Figshare are also assigned a Direct Object Identifier (DOI) link, which helps Google discover them in searches, such as those searches conducted by researchers performing literature reviews in the future. These Figshare poster publications can then link to video talks on YouTube, Youku, etc. and extended abstracts for more detail.

\section{Abstracts can be made more accessible as PrePrints}

Before creating their talk or poster, some fields ask scientists to submit 5-15 page extended abstracts containing details about the new research they would like to present. If these essays pass peer review, then the author is given permission to present them as a talk or poster. But what happens to the essay?

Right now, abstract essays are often left to rot behind paywalls and loginwalls, only discoverable by using the conference's limited, members-only search feature. These conference abstracts include details and references typically not found in the talk or poster, and can be incredibly helpful to scientists who are conducting literature reviews in the future.

For other fields, posters and presentations communicate later-stage research that is already in the process of being published, which often means that draft manuscripts of the research exist.

Requiring conference presenters to upload their extended abstracts or pre-published manuscripts to a PrePrint website (e.g., bioRxiv.org) prior to submission would ensure that all conference abstracts and associated manuscripts are available Open Access and discoverable via Google Scholar and similar search engines. 


\section{Social media posts can teach, not just tantalize.}

Social media platforms (e.g., Twitter, Tiktok, Instagram) can dramatically increase the impact and reach of all forms of scientific content, and may drive more member registrations and profits to the associated conferences.

\section{Social media statements are good advertising}

Already, innovative scientific conferences will ask scientists to submit a 'social media statement' to be posted from the conference's social media accounts. Here is a real example submitted by our first author: "Want to find out how people talk about their work when they find it meaningful? Come see our \#SIOP19 poster at 2pm on Tuesday, board \#252!" These social media statements help get the word out, but typically do not contain any actual insight; they merely promise to teach the reader insight if they attend.

\section{Social media 'microlearning' posts actively teach new science}

The biggest leap forward in impact we can make when sharing science on social media is to use it for more than advertising — to actively teach people new research in bite-sized posts, and link those mini-lessons to articles and talks for even deeper learning.

Instead of a purely 'promotional' Tweet like the above example, the author could include a key takeaway: "When people find their work meaningful, they talk about their work like it is part of their identity." and then link people to the extended abstract PrePrint, presentation video, and the conference event schedule for more.

Teaching people something valuable about a study in the span of a Tweet or Instagram post puts off a stronger information scent (Pirolli \& Card, 1995). It establishes that Tweet as providing a high rate of reward for a small investment of attention. Counterintuitively, this may actually provide stronger bait and make people more likely to click through to the talk or article. At minimum, everyone who reads the Tweet learns something, whether they click or not.

\section{Social media mini-posters are easy to make and highly engaging}

To maximize the engagement with science posted on social media, we can go beyond text-only posts and create social media 'flipbook' mini-posters (see Figure 2). Essentially a silent presentation, these flipbooks can be 3-5 Powerpoint slides illustrating the key aspects of a study in a visual format. When posted on social media, the slides either auto-advance or allow scientists to swipe through them at their own pace. 


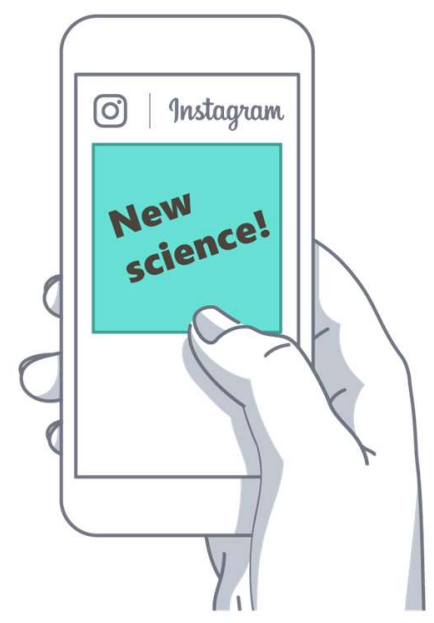

1. A new finding appears in this scientist's Instagram feed.

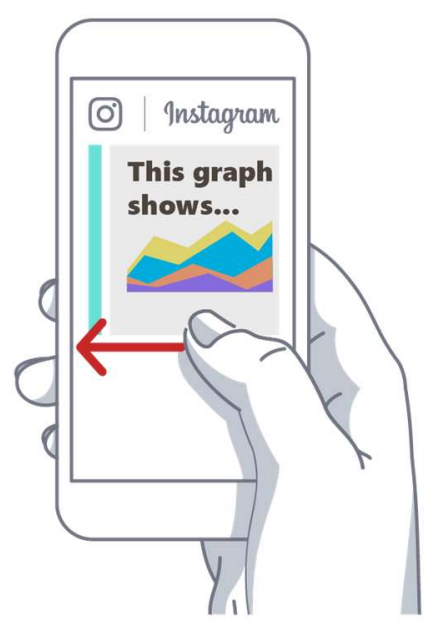

2. They swipe-left to learn the key points of the study in 3-5 mini slides.

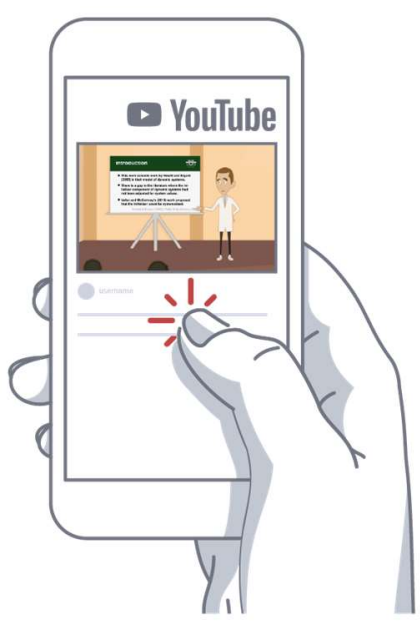

3. Then click to watch the talk, which links to the Open Access PrePrint.

Figure 2. Social media 'flipbook' mini posters are 3-5 slides teaching the key points of a research study that can be created in PowerPoint, automatically posted to social media, and linked to the conference talk video or PrePrint essay.

Examples of researchers using this technique to share new science can be seen across many fields under the "\#TwitterPoster" hashtag on Twitter (also see Morrison, 2020). As one prominent example, graduate students in the Psychology department at University College Dublin organized their own unofficial social media poster session full of these flipbook-style mini posters that was so popular that their virtual poster session became a trending hashtag (Twitter hashtag search, 2020).

This same 'flipbook' design pattern would also probably make an excellent virtual poster experience apart from social media.

\section{Focusing on user goals can help conferences invent all-new formats}

Now that conferences are online, any format that can be imagined for scientific knowledge exchange can be realized with the right technical resources, including holding an entire conference in Virtual Reality, as the 2020 Educators in VR International Summit chose to do (Vanfossen, 2020). 
The technical possibilities on the web are endless, but the goals of scientists who attend conferences are more finite. Here are a few of them:

1. Get updated on my focal area.

2. Learn about a new area I'm interested in.

3. See what's going on in my field broadly (give serendipity a chance).

4. Meet new people in my field.

It can help to focus less on translating traditional formats, and more on translating traditional goals. For example, the Van Andel Institute (2020) met the social goals of a poster session by creating a chatroom-based poster session using Slack. Similarly, the SABER community asked presenters to post 1-minute personal summary videos next to each poster file (SABER Community, 2020).

To help researchers get updated on their focal area, conferences could use the data they already have on which topics each researcher has presented on, and create short 'new findings in your topic area' pages tailored to the individual.

\section{Motivating scientists to try new formats}

How can conferences motivate busy scientists to create social media mini-posters, put our talks on YouTube, upload our abstracts as preprints, and use all-new formats? Easy: Just nudge us to do it at exactly the right time - when we need something from the conference.

\section{Use the Goal Gradient}

Scientists are typically too busy to volunteer extra effort in the name of impact, except when we are in the process of submitting to a conference. In that frantic state, we will jump through any hoop to get our work submitted. This is called the Goal Gradient hypothesis: The closer someone is to their goal, the more effort they will put in (Kivetz, Urminsky, \& Zheng, 2006).

The Goal Gradient hypothesis suggests that we have a lot of leeway in what we can ask scientists to do at the submission stage. In our view, much of the above (e.g., asking for links to PrePrints of extended abstracts and manuscripts), would work well as requirements added to the process of submitting to a conference.

\section{Send impact reports to give scientists more meaning}

It could be incredibly motivating to send scientists impact reports to help them see that, contrary to their expectations, people actually downloaded their posters and viewed their talks. To take this up to the level of the whole field, the 'total views' for all content in the conference across all 
platforms could become a field-wide impact metric that can be tracked and celebrated year-overyear.

\section{Maintaining value for paying members with drip-release}

A field's annual conference is a crucial source of income for many scientific societies, which often motivates the 'paywall model' for online conference content. One antidote to this is a 'driprelease' model, where all content is made immediately available to paying members, but is then scheduled to 'drip' out to public repositories and social media over the next year.

The world-renowned TED (Technology Entertainment Design) conference uses exactly this type of drip-release model. They publicly release one talk per day on YouTube (and their public website) over the year following their in-person conference, accompanied by social media posts with key takeaways (McManus, 2013).

This content drip can be automated, so that a year's worth of YouTube uploads and social media posts can be put on autopilot with one, initial burst of effort. The content-drip keeps dues-paying members engaged and learning throughout the year, and builds interest and excitement for next year's conference. It also strengthens the conference's brand image as a source of knowledge in that area.

\section{Overcoming objections}

Many scientists are uncomfortable with the idea of making scientific content - especially conference content - more broadly accessible.

Objection \#1: "The public won't understand that conference research is early-stage." There is an assumption that scientists always critically evaluate conference research for its 'potential', whereas the naive, gullible public will accept everything we say as gospel and immediately start bottling it into snake oil.

Remember that our current approach (where the content disappears when the conference ends) is keeping new science away from many experts who would understand and benefit from it (for example, scientists in the field who could not attend that particular conference). Is it worth keeping new science away from those people to coddle the public? The best long-term outcome is not keeping the public blind to raw science; it is teaching them to understand it. And if we want the public to better understand science, then at some point we cannot keep protecting them from it. 


\section{Objection \#2: "We shouldn't dumb it down for social media."}

When worrying that a complex, nuanced study cannot possibly be boiled down to a Tweet or a Reddit post, it is important to note that science is already distilled successfully on Social media on a large scale. The "reddit.com/r/science" community posts single-sentence summaries of new findings (linked to study abstracts) for an audience of over 24 million subscribers.

Sometimes a finding on reddit.com/r/science summary too over-simplified (e.g., neglects to say "in mice"), and in those cases the top comment (often from a scientist in a related field) will typically correct the misimpression and provide the missing context.

Websites like reddit and Twitter are populated by a mix of scientists and lay-people, and are already capable of doing a pretty good job of summarizing new science. They can only improve if authors more actively shape the interpretation of their own work.

\section{Objection \#3: "I just don't feel confident enough in my presenting ability."}

Our first and third authors have pressed many scientists to share their work more broadly. Often, after getting past the above objections, these researchers confessed something to the effect of "I just don't feel confident enough in my presenting ability to want people to see my talks and posters." Happily, some of these same scientists have shown renewed interest in sharing their talks more widely after giving a talk they are especially proud of.

If we can build more self-efficacy in scientists about communicating their work, the rest of the objections may fall, as research in Work Psychology has shown that feeling like you are good at something makes you more interested in doing it (Zimmerman \& Wiernik, 2020).

\section{Conclusion: Every product of a scientific conference can be made available free, online, and easy to browse.}

If the above practices are implemented, it will result in tens of thousands of new scientific talks, posters, and papers flooding the public internet and social media every year. Every scientist will be able to quickly access any content from any conference that could aid their research (without needing to log-in or request copies from authors). And millions of people searching public video sites like YouTube and Youku to learn about a new topic will begin discovering real science on it.

Making science more easily accessible to the world will not be without some negative consequences, but it could also accelerate the pace of discovery and build a direct line of communication between science and the public. And all it takes to start this revolution is a few clicks of an upload button. 


\section{References}

Consumer Electronics Show (2019). Consumer Technology Association Attendance Audit Summary. https://cdn.ces.tech/ces/media/pdfs/ces-2019-audit-summary.pdf

Kivetz, R., Urminsky, O., \& Zheng, Y. (2006). The goal-gradient hypothesis resurrected: Purchase acceleration, illusionary goal progress, and customer retention. Journal of Marketing Research, 43(1), 39-58.

McManus, E. (2013). How long does it take for a TED talk to show up as a publicly viewable video? [Online forum poster]. Quora. https://www.quora.com/How-long-does-it-take-fora-TED-talk-to-show-up-as-a-publicly-viewable-video.

Merlo, K. L., Wossner, Z., \& Morrison, M. A. (2019). \#BetterPoster at the 2019 American Heart Association Scientific Sessions. [Report].

Morrison, M. A. (2020, March 24). How to create a quick Twitter Poster to share new research (includes templates).[YouTube Video]. https://youtu.be/fQDL8r3r_d4.

Pirolli, P., \& Card, S. (1999). Information foraging. Psychological Review, 106(4), 643-675. SABER. (2020, July 17). SABER virtual poster session [Twitter status]. Retrieved from https://twitter.com/SABERcommunity/status/1284225713676132353

SimilarWeb (2020, June). Traffic Overview for Youtube.com. https://www.similarweb.com/website/youtube.com/

Twitter (2020). Hashtag search \#GIFSFromYourGaff. https://twitter.com/hashtag/GIFsFromYourGaff

Van Andel Institute (2020). Posters. https://originsofcancer.org/posters/

Vanfossen, L. (2020, March 8). Lessons learned from hosting a VR conference. Educators in VR. https://educatorsinvr.com/2020/03/08/behind-the-scenes-of-the-educators-in-vrinternational-summit/] Zimmerman, M.D., \& Wiernik, B.M. (2020, April 25). Interests and self-efficacy: One construct or "Related but distinct"? [Poster Presentation]. Society for Industrial and Organizational Psychology conference, Austin, Texas, USA. 